\title{
THE ROLE OF THE DISCRETE-TIME KALMAN-YAKUBOVITCH-POPOV LEMMA IN DESIGNING STATISTICALLY OPTIMUM FIR ORTHONORMAL FILTER BANKS ${ }^{\dagger}$
}

\author{
Jamal Tuqan and P. P. Vaidyanathan \\ Department of Electrical Engineering 136-93 \\ California Institute of Technology, Pasadena, CA 91125, USA. \\ tuqan@systems.caltech.edu, ppvnath@sys.caltech.edu
}

\begin{abstract}
We introduce a new approach to design FIR energy compaction filters of arbitrary order $N$. The optimization of such filters is important due to their close connection to the design of an $M$-channel orthonormal filter bank adapted to the input signal statistics. The novel procedure finds the optimum product filter $F_{o p t}(z)=H_{o p t}(z) H_{o p t}\left(z^{-1}\right)$ corresponding to the compaction filter $H_{\text {opt }}(z)$. The idea is to express $F(z)$ as $D(z)+D\left(z^{-1}\right)$ and reformulate the compaction problem in terms of the state space realization of the causal function $D(z)$. For a fixed input power spectrum, the resulting filter $F_{\text {opt }}(z)$ is guaranteed to be a global optimum due to the convexity of the new formulation. The new design method can be solved quite efficiently and with great accuracy using recently developed interior point methods and is extremely general in the sense that it works for any chosen $M$ and any arbitrary filter length $N$. Finally, obtaining $H_{\text {opt }}(z)$ from $F_{\text {opt }}(z)$ does not require an additional spectral factorization step. The minimum phase spectral factor can be obtained automatically by relating the state space realization of $D_{o p t}(z)$ to that of $H_{o p t}(z)$.
\end{abstract}

\section{INTRODUCTION}

Consider the scheme of Fig. 1 where $H(z)$ is a real coefficient FIR filter of order $N$ and $x(n)$ is a zero mean WSS random process with a fixed power spectrum $S_{x x}\left(e^{j \omega}\right)$. The output of the filter is decimated by $M$ to produce $y(n)$. For a fixed pair $(M, N)$, the FIR energy compaction problem is the following:

$$
\max _{H\left(e^{j \omega}\right)} \int_{-\pi}^{\pi}\left|H\left(e^{j \omega}\right)\right|^{2} S_{x x}\left(e^{j \omega}\right) \frac{d \omega}{2 \pi}
$$

subject to

$$
\left.\frac{1}{M} \sum_{k=0}^{M-1} \mid H\left(e^{j(\omega-2 \pi k / M)}\right)\right)\left.\right|^{2}=\left.\left|H\left(e^{j \omega}\right)\right|^{2}\right|_{\downarrow M}=1
$$

The constraint (2) means that the magnitude squared response $\left|H\left(e^{j \omega}\right)\right|^{2}$ is Nyquist $(M)[1$, pages $151-152]$.

The design of FIR energy compaction filters has received considerable attention due in part, to the fact that they

\footnotetext{
$\dagger$ This work is supported in parts by the NSF grant 0703755
} and Tektronix, Inc. are the building blocks in the design of orthonormal filter banks when subband quantizers are present. Given a fixed budget of $b$ bits for the subband quantizers, the design of an optimum orthonormal filter bank consists of simultaneously optimizing the analysis and synthesis filters as well as choosing a subband bit allocation strategy such that the average variance of the output quantization noise is minimized. Under the high bit rate quantizer assumptions and with the optimum bit allocation, the objective function is the well known coding gain expression:

$$
\mathcal{G}_{S B C}(M)=\frac{\sigma_{x}^{2}}{\left(\prod_{k=0}^{M-1} \sigma_{x_{k}}^{2}\right)^{1 / M}}
$$

where $\sigma_{x_{k}}^{2}$ is the variance of the $k$ th subband signal.

For the FIR two-channel case $(M=2)$, maximizing the coding gain is equivalent to the FIR energy compaction problem [2]. For the FIR $M$-channel case, the analysis filters are optimized to minimize the geometric mean of the subband variances under the orthonormality condition. The geometric mean is a concave function making the problem a difficult one. An alternative solution to the direct minimization of the geometric mean is the design of a socalled principal component filter bank (PCFB). A PCFB is defined as follows [3]: consider Fig. 2 where $(M-P)$ channels are dropped in the synthesis part of an $M$-channel orthonormal filter bank. An orthonormal filter bank that minimizes the average mean square reconstruction error for all $P$ is termed a principal component filter bank (PCFB). From the orthonormality property, a PCFB therefore generates a decreasing arrangement of the subband variances $\sigma_{x_{1}}^{2} \geq \sigma_{x_{2}}^{2} \ldots \geq \sigma_{x_{P}}^{2}$ such that, for all $1 \leq P<M, \sum_{k=1}^{P} \sigma_{x_{k}}^{2}$ is maximized. For $P=M$, the sum of the subband variances is fixed and is equal to $M \sigma_{x}^{2}$. The set of subband variances $\left\{\sigma_{x_{k}}^{2}\right\}$ produced by a PCFB is said to "majorize" any other arbitrary set of subband variances $\left\{\sigma_{y_{k}}^{2}\right\}$. Using a standard theorem $[4,10$, pages 199-200], the majorization property of the subband variances of a PCFB implies, in particular, that $\left(\prod_{k=0}^{M-1} \sigma_{x_{k}}^{2}\right)^{1 / M}$ is minimized. Unfortunately, the existence of a PCFB is guaranteed for only two extreme cases: $N<M$ (the KLT solution) and $N=\infty$ (the ideal filter solution [3]). Nevertheless, if a PCFB exits, then, designing an optimal FIR energy compaction filter is a necessary first step in finding such a filter bank [5]. 


\section{THE PRODUCT FILTER FORMULATION}

From (1) and (2), we can immediately observe that the optimum solution, if it exists, is only a function of $\left|H\left(e^{j \omega}\right)\right|^{2}$. By denoting the product filter $H(z) H\left(z^{-1}\right)$ by $F(z)$, the output variance $\sigma_{y}^{2}$ in (1) can be rewritten as

$$
\sigma_{y}^{2}=r(0)+2 \sum_{n=1}^{N} f(n) r(n)
$$

and the constraint (2) becomes

$$
\begin{gathered}
f(M n)=\delta(n) \\
F\left(e^{j \omega^{\prime}}\right) \geq 0 \quad \forall \omega
\end{gathered}
$$

where $r(i)$ denotes the $i^{\text {th }}$ autocorrelation coefficient of the input $x(n)$. The problem is now linear in the optimization variables $f(n), n \geq 1$ at the expense of an additional constraint, namely equation (6) referred to as the positivity constraint. The positivity constraint has to be satisfied at each frequency $\omega$ and is therefore equivalent to an infinite number of inequality constraints. The above formulation has a finite number of variables and an infinite number of constraints, hence the name semi-infinite programming (SIP). The semi-infinite program can be approximated by sampling or discretizing the continuous frequency axis. We choose a finite set of discrete frequencies $\left\{\omega_{i}, 0 \leq i \leq L\right\}$, often uniformly spaced, and enforce the positivity constraint only at those frequencies. This approach was first suggested and analyzed in depth by Moulin et al. [6]. Other discretization methods can be also found in $[7,8]$. The main problem with the sampling approach is that we can no longer guarantee the positivity of $F_{o p t}(z)$ between the discrete frequencies, no matter how large $L$ is. This, in turn, creates an infeasible spectral factorization step. Indeed, the discretized version is an outer approximation of the original SIP problem; its feasible set includes the feasible set of the original SIP problem. We show next, using the discrete-time Kalman-Yakubovitch-Popov lemma, that (6) can be satisfied over all $\omega$ at the expense of $N(N+1) / 2$ additional variables.

\section{THE DISCRE'TE TIME KYP LEMMA}

Since $F(z)=H(z) H\left(z^{-1}\right)$, the product filter is a two sided symmetric sequence and we can therefore write $F(z)$ as $D(z)+D\left(z^{-1}\right)$. The causal function $D(z)$ completely characterizes $F(z)$ and it is natural to wonder whether the positivity condition on $F\left(e^{j \omega}\right)$ can be reformulated in terms of some other condition(s) on $D\left(e^{j \omega}\right)$. The answer turns out to be yes and is established by the well known discrete time positive real lemma (KYP lemma) [9]. We start with this preliminary lemma.

Lemma 1. Discrete time positive real functions [9]. A square transfer matrix (function) $D(z)$ whose elements are real rational functions analytic in $|z|>1$ is discrete positive real if, and only if, it satisfies all the following conditions :

$$
\begin{array}{r}
\text { poles of } D(z) \text { on }|z|=1 \text { are simple } \\
D\left(e^{j \omega}\right)+D\left(e^{-j \omega}\right) \geq 0 \quad \forall \omega \text { at which } D\left(e^{j \omega}\right) \text { exists }
\end{array}
$$

Furthermore, if $z_{0}=e^{j \omega_{0}}, \omega_{0}$ real, is a pole of $D(z)$ and if $\mathbf{K}$ is the residue matrix of $D(z)$ at $z=z_{0}$, the matrix $Q=e^{-j \omega_{0}} \mathbf{K}$ is hermitian positive semi definite.
Assume now that $D(z)$ has the following state space realization :

$$
\begin{aligned}
x(n+1) & =A_{d} x(n)+B_{d} u(n) \\
y(n) & =C_{d} x(n)+D_{d} u(n)
\end{aligned}
$$

where $A_{d}$ is $N \times N, B_{d}$ is $N \times P, C_{d}$ is $L \times N$, and $D_{d}$ is $L \times P$. For our case, $P=L=1$. Then, the following lemma can be established.

Lemma 2. The discrete time KYP lemma [9]. Let $D(z)$ be a transfer matrix (function) with real rational elements that is analytic in $|z|>1$ with only simple poles on $|z|=$ 1. Let $\left(A_{d}, B_{d}, C_{d}, D_{d}\right)$ be a minimal realization of $D(z)$. Then, $D(z)$ is discrete positive real if, and only if, there exist a real symmetric positive definite matrix $P_{d}$ and real matrices $W_{d}$ and $L_{d}$ such that :

$$
\begin{aligned}
P_{d}-A_{d}^{T} P_{d} A_{d} & =L_{d}^{T} L_{d} \\
C_{d}^{T}-A_{d}^{T} P_{d} B_{d} & =L_{d}^{T} W_{d}^{T} \\
D_{d}+D_{d}^{T}-B_{d}^{T} P_{d} B_{d} & =W_{d}^{T} W_{d}
\end{aligned}
$$

The above equalities (10-12) can be rewritten as the following matrix inequality :

$$
\left[\begin{array}{cc}
P_{d}-A_{d}^{T} P_{d} A_{d} & C_{d}^{T}-A_{d}^{T} P_{d} B_{d} \\
C_{d}-B_{d}^{T} P_{d} A_{d} & D_{d}+D_{d}^{T}-B_{d}^{T} P_{d} B_{d}
\end{array}\right] \succeq 0
$$

where the notation $\succeq$ indicates that the above matrix should be positive semi-definite. Equation (13) represents therefore an equivalent condition for the positivity constraint. Assume now that $D(z)$ is implemented in a direct form structure with the following state space representation:

$$
\begin{aligned}
& A_{d}=\left[\begin{array}{cc}
0 & I \\
0 & 0
\end{array}\right], \quad B_{d}=\left[\begin{array}{c}
0 \\
0 \\
\vdots \\
1
\end{array}\right] \\
& C_{d}=[f(N) \ldots f(1)], \quad D_{d}=\frac{1}{2}
\end{aligned}
$$

Clearly, this state space realization is minimal since the number of delay elements is equal to the degree of $D(z)$. Then, the objective function becomes:

$$
C_{d} \mathbf{R}^{T}
$$

where $\mathbf{R}^{T}=[r(N) \ldots r(1)]^{T}$ and the Nyquist constraint can be written as a linear equality constraint:

$$
Q C_{d}^{T}=0
$$

where 0 is the zero vector and $Q$ is a diagonal matrix with diagonal elements $\in\{0,1\}$. The positions of the unity elements are determined by $N$ and $M$. For example, for $N=5$ and $M=2$, the diagonal elements are $\left\{\begin{array}{lllll}0 & 1 & 0 & 1 & 0\end{array}\right\}$. Summarizing, we can represent the positivity constraint as a "linear" matrix inequality (LMI) whose entries are affine functions of the variables $P_{d}$ and $C_{d}$, and the Nyquist constraint as an equality constraint on $C_{d}$.

It is important to notice the differences between several variations of the same problem. The FIR energy compaction problem, expressed in terms of the filter $H(z)$, is a non linear non convex optimization problem. The product 
filter formulation is a semi-infinite, linear and convex problem. The discretized product filter formulation is linear, convex, and can be solved using standard linear programming problem but is an approximation of the original SIP. The state space formulation, proposed in this paper, is non linear, convex and semi-definite. Using the rationality of $F(z)$, the infinite set of inequality constraints are replaced by a (finite dimensional) positive semi definite constraint (13) with the auxiliary variable $P$, permitting the theoretical solution to be found.

The optimization problem described by (15), (13) and (16) is a maximization problem in the in the variable vector $C_{d}$ and a feasibility problem in the matrix $P_{d}$. In principle, we can obtain a global optimum $C_{d_{o p t}}$ and a feasible matrix $P_{d}$ and, then, spectral factorize $F_{o p t}(z)$ to obtain $H_{o p t}(z)$. It turns out however that the spectral factorization step can be completely avoided by writing the state space representation of the minimum phase spectral factor, $H_{\min }(z)$, in terms of the matrices $A_{d}, B_{d}, C_{d}, D_{d}$ and a particular $P_{d}$, namely the minimum element $P_{d_{\min }}$ of the convex set of positive definite matrices satisfying equation (13) and (16). This is established in the next section.

\section{THE MINIMUM PHASE SPECTRAL FACTOR}

Definition. [10, pages 469-476] Minimum element. We say that $P_{d_{\text {min }}} \in S$ is a minimum element of $S$ with respect to the (strict) generalized inequality $\preceq(\prec)$ if for every $P \in$ $S$ we have $P_{d_{\min }} \preceq(\prec) P$. Note that $P_{d_{2}} \succeq P_{d_{1}}$ is equivalent to $P_{d_{2}}-P_{d_{1}}$ is positive semi definite. If a set has a minimum element, this element is unique.

Theorem. [11] Assume that $D(z)$ satisfies the discrete time KYP lemma with a minimal realization $\left(A_{d}, B_{d}, C_{d}\right.$, $\left.D_{d}\right)$. In particular, $F\left(e^{j \omega}\right)=D\left(e^{j \omega}\right)+D\left(e^{-j \omega}\right) \geq 0 \forall \omega$. Then, the minimum phase spectral factor $H_{\min }(z)$ of $F(z)$ can be expressed in the form:

$$
H_{\min }(z)=W_{d}+L_{d}^{T}\left(z I-A_{d}\right)^{-1} B_{d}
$$

where

$$
\begin{aligned}
W_{d} & =\left(D_{d}+D_{d}^{T}-B_{d}^{T} P_{\min } B_{d}\right)^{1 / 2} \\
L_{d}^{T} & =\frac{\left(C_{d}^{T}-A_{d}^{T} P_{\min } B_{d}\right)}{\left(D_{d}+D_{d}^{T}-B_{d}^{T} P_{\min } B_{d}\right)^{1 / 2}}
\end{aligned}
$$

and $P_{d_{m i n}}$ is the minimum element in the convex set of symmetric positive definite matrices satisfying the LMI (13) and the Nyquist constraint (16).

Corollary. [11] Assume that $D(z)$ is implemented in a direct form structure with the state space representation given in (14). The minimum phase spectral factor $H_{\min }(z)$ of $F(z)$ is then given by:

$$
\begin{aligned}
H_{\min }(z) & =\frac{1}{\sqrt{1-p_{d_{\min }}(N, N)}}\left\{1-p_{d_{\min }}(N, N)\right. \\
& +\left(f(1)-p_{d_{\min }}(N-1, N)\right) z^{-1} \\
& +\ldots-p_{d_{\min }}(N-M, N) z^{-M} \\
& \left.+\ldots+f(N) z^{-N}\right\}
\end{aligned}
$$

Since $A_{d}, B_{d}$ and $D_{d}$ are already fixed by the choice (14), $H_{\min }(z)$ is automatically obtained once the program returns $C_{d}$ and $P_{d_{\min }}$. We can include $P_{d}$ in the objective function (15) but minimizing $P_{d}$ directly will produce a vector valued objective function. Instead, we minimize a scalar valued function of $P_{d}$ using the following observation.

Observation 1. Assume that $P_{d_{m i n}}$ is the minimum element in the convex set of symmetric positive definite matrices satisfying the LMI and Nyquist constraints (13) and (16). Then, $P_{d}=P_{d_{\min }}$ if and only if $\operatorname{Tr}\left(\mathrm{W} P_{d}\right)$ is minimum for every diagonal positive semi-definite matrix $\mathbf{W}$.

\section{THE FINAL FORMULATION}

The optimization problem reduces to the following final form:

$$
\max _{C_{d}, P_{d}} C_{d} \mathbf{R}^{T}-\operatorname{Tr}\left(\mathbf{W} P_{d}\right)
$$

where $\mathbf{R}^{T}=[r(N) \ldots r(1)]^{T}$ and $\mathbf{W}$ is a diagonal positive semi definite weight matrix subject to

$$
\begin{aligned}
& {\left[\begin{array}{cc}
P_{d}-A_{d}^{T} P_{d} A_{d} & C_{d}^{T}-A_{d}^{T} P_{d} B_{d} \\
C_{d}-B_{d}^{T} P_{d} A_{d} & D_{d}+D_{d}^{T}-B_{d}^{T} P_{d} B_{d}
\end{array}\right] \succeq 0} \\
& Q C_{d}^{T}=0
\end{aligned}
$$

This final formulation is therefore a maximization problem in the variable vector $C_{d}$ and a minimization problem in the matrix $P_{d}$.

Observation 2. The optimization problem (21), (22) and (23) is a convex program in the variables $C_{d}$ and $P_{d}$.

The above formulation is therefore a convex multi-objective optimization problem for which any local solution is also a global one. The weight matrix $\mathbf{W}$ is included in the objective function (21) because, unlike the formulation in section 3 , we now have two competing objectives. The idea is to choose the weight matrix so that optimality of $C_{d}$ is never compromised, i.e., in order to prohibit $\operatorname{Tr}\left(\mathbf{W} P_{d}\right)$ from becoming the dominant factor in (21). The particular choice of the trace function was intentional in order to use semi definite programming. Semi definite programs can be solved very efficiently both in theory and practice [12]. Two different programs are currently available at our web cite: the first one is written by Vandenberghe and Boyd [13] and uses a particular primal-dual interior point method. The second one uses the MATLAB LMI toolbox that implements the projective algorithm of Nesterov and Nemirovskii [14]. For more details, the reader is referred to [11] and our home page at http://www.systems.caltech.edu/tuqan.

\section{EXAMPLE}

Multiband AR(5) process. Assume that the input $x(n)$ is a zero mean AR(5) process with a multiband power spectrum (dashed curve in Fig. 3). The magnitude squared responses $\left|F_{\text {opt }}\left(e^{j \omega}\right)\right|^{2}$ for $N=7,17$ and 27 and $M=3$ are shown in Fig. 3. The compaction gains are 1.867, 2.007 and 2.045 respectively. Note that the compaction gain is upper bounded by $M=3$. We have chosen to set $\mathbf{W}=\alpha \mathbf{I}$ and $\alpha=10^{-6}$. Although there is no formal proof that this particular choice of $\mathbf{W}$ will work for all inputs, we never had to change this setting over all the examples we have tried. The reader is referred to $[2,11]$ for more numerical results. 


\section{CONCLUDING REMARKS}

Although the emphasis of this paper is on the design of FIR energy compaction filters and its connection to optimum FIR orthonormal filter banks, the newly proposed method is general enough to cover a, wide variety of signal processing applications. One such application is the standard problem of designing FIR orthonorjonal filter banks where the filters have minimum stopband energy. Another important application is the problem of designing optimum FIR transmitter and receiver filters for data transmission over bandlimited channels [15]. The filters are designed so that optimum spectral concentration is achieved in the transmission bandwidth of the channel, and zero intersymbol interference (ISI) is obtained when the filters operate in cascade. By replacing $S_{x x}\left(e^{j \omega}\right)$ with $\operatorname{rect}\left(\omega / \omega_{c}\right)$, where $\omega_{\mathrm{c}}$ is the cut off frequency of the low pass channel and with a receiver filter $H_{r}\left(e^{j \omega}\right)$ matched to the transmitter filter $H_{t}\left(e^{j \omega}\right)$, i.e., $H_{r}\left(e^{j \omega}\right)=H_{t}^{*}\left(e^{j \omega}\right)$ where $*$ denotes complex conjugation, the problem can be expressed in the form described by (1) and (2). Other applications are in echo cancelation [16], multirate signal modeling [17] and optimization of wavelet basis $[3,18]$ to name a few.

\section{REFERENCES}

[1] P. P. Vaidyanathan, Multirate systems and filter banks. Englewood Cliffs, NJ: Prentice Hall, 1993.

[2] J. Tuqan and P. P. Vaidyanathan, "Globally optimal two-channel FIR orthonormal filter banks adapted to the input signal statistics," ICASSP Proc., May 1998.

[3] M. K. Tsatsanis and G. B. Giannakis, "Principal component filter banks for optimal multiresolution analysis," IEEE Trans. on SP, pp. 1766-1777, Aug. 1995.

[4] A. W. Marshall and I. Olkin, Inequalities : Theory of majorization and its applications. Acad. Press, 1979.

[5] P. Moulin and M. K. W. Mihcak, "Theory and design of signal adapted FIR paraunitary filter banks," IEEE transactions on Signal Processing, April 1998.

[6] P. Moulin, M. Anitescu, K. Kortanek, and F. A. Potra, "The role of linear Semi-Infinite programming in signal dapted QMF bank design," IEEE transactions on Signal Processing, vol. 45, pp. 2160-2174, Sep. 1997.

[7] J.-C. Pesquet and P. L. Combettes, "Wavelet synthesis by alternating projections," IEEE Trans. on Signal Processing, vol. 44, pp. 728-732, March 1996.

[8] A. Kirac and P. P. Vaidyanathan, "FIR compaction filters : new design methods and properties," in ICASSP Proc., vol. 3, pp. 2229-2232, 1997.

[9] B. E. L. Hitz and B. D. O. Anderson, "Discrete positive-real functions and their application to system stability," Proc. IEE, pp. 153-155, January 1969.

[10] R. A. Horn and C. R. Johnson, Matrix analysis. 1985.

[11] J. Tuqan and P. P. Vaidyanathan, "A state space approach to the design of globally optimum FIR energy compaction filters," Technical report, Caltech, 1998.

[12] L. Vandenberghe and S. Boyd, "Semidefinite programming," Siam review, vol. 38, pp. 49-95, March 1996.
[13] L. Vandenberghe and S. Boyd, "SP: software for semi definite programming," October 1994.

[14] Y. Nesterov and A. Nemirovskii, Interior point polynomial algorithms in convex programming. vol. 13 of studies in applied mathematics, SIAM, phi., PA, 1994.

[15] P. R. Chevillat and G. Ungerboeck, "Optimum FIR transmitter and receiver filters for data transmission over band-limited channels," IEEE Trans. on Communications, vol. 30, pp. 1909-1915, Aug. 1982.

[16] Q. Jin, Z.-Q. Luo, and K. M. Wong, "Optimum filter banks for signal decomposition and its applications in adaptive echo cancellation," IEEE Trans. on Signal Processing, vol. 44, pp. 1669-1689, July 1996.

[17] J. Tuqan and P. P. Vaidyanathan, "The design of optimum filters for quantizing a class of non bandlimited signals," ISCAS Proc., June 1998.

[18] H. Caglar, Y. Liu, and A. N. Akansu, "Statistically optimized PR-QMF design," in SPIE, Visual Comm. and Image Proc. '91, vol. 1605, pp. 86-94, 1991.

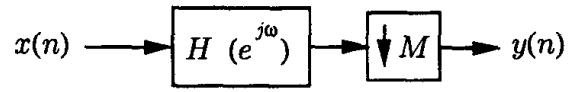

Figure 1: Schematic of the FIR energy compaction problem.

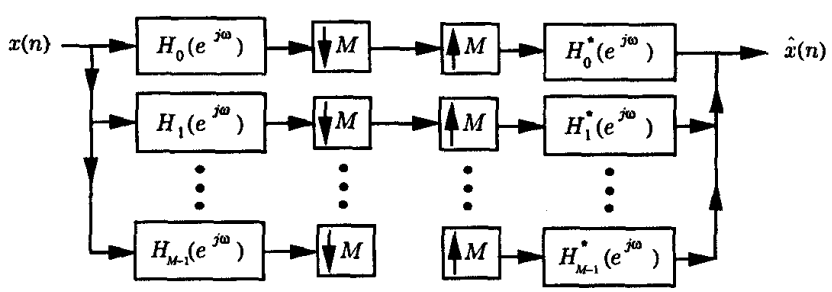

Figure 2: An $M$-channel FIR principal component filter bank where only the first two channels are retained

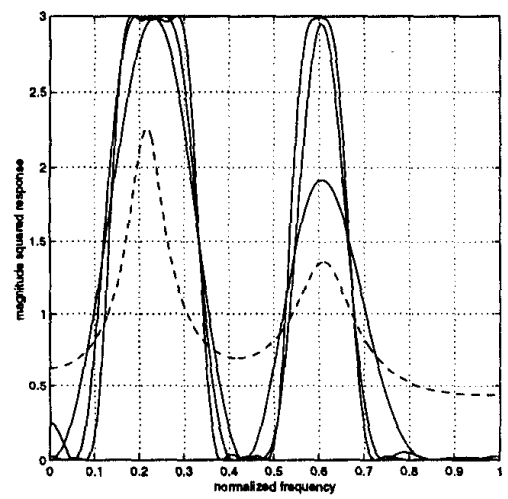

Figure 3: The magnitude squared responses of the optimum compaction filters corresponding to the multiband $\mathrm{AR}(5)$ process of order $N=7,17$ and 27 with $M=3$. 